\title{
Strategy choice and the effect of field independence on abstraction, storage, and retrieval
}

\author{
JUDITH E. HENNESSEY and IRWIN D. NAHINSKY \\ University of Louisville, Louisville, Kentucky 40208
}

\begin{abstract}
Subjects were presented a concept identification (CI) task in which stimuli were varied on four dimensions descriptive of people. Half of the stimuli contained names and pictures of well-known people exemplifying the descriptive dimensions. Upon completion of the CI problem, subjects were given a surprise recognition memory task for CI stimuli in which they had to discriminate between old and new stimuli differentiated only by the exemplars that accompanied verbal descriptions. Subjects were scored for the degree to which they accessed stimuli with pictorial exemplars faster than those without them in the recognition task. For subjects whose latencies were not influenced by the pictorial variable, efficiency in $\mathrm{CI}$ and accuracy of recognition were positively related to scores on the embedded figures test. It was concluded that field articulation was related to superior ability to process information in a working memory when an analytic processing strategy is used.
\end{abstract}

Recent years have witnessed an attempt to apply the methods of cognitive psychology to understand the nature of individual differences in abilities revealed by more traditional psychometric methods. Many of these differences no doubt represent differential capacity limitations for processing information. One obstacle in using processing tasks to study capacity differences involves strategies that might be applied to such tasks. Control processes related to overall strategies tend to be labile and subject to varied experience, whereas automated or "lower level" processes tend to be stable. A study of cognitive processes underlying interindividual difference variables must separate strategy variables from those associated with more primitive processes in order to develop understanding of the nature of capacity differences. The work of MacLeod, Hunt, and Mathews (1978) exemplifies an attempt to separate strategy and capacity variables in the domain of verbal ability.

Witkin, Dyk, Faterson, Goodenough, and Karp (1962) found that the variable of perceptual field dependence was consistently related to a number of important aspects of cognitive functioning. Field independence may be defined as an ability to "break up" a configuration and abstract relevant features from an embedding context. It has been suggested that this ability reflects the operation of a certain "cognitive style" in information processing that determines an

This investigation was supported in part by the U.S. Army Research Institute for the Behavioral and Social Sciences, Grant DAHC 19-74-G-0007 to the second author. Requests for reprints should be addressed to Irwin D. Nahinsky, Department of Psychology, University of Louisville, Louisville, Kentucky 40208. individual's approach to tasks rather than the efficiency of his/her performance.

The process of abstraction, whereby concepts are learned inductively and represented in memory, seems to be an area that would naturally lend itself to a study of the disembedding process. The tendency of an individual to process a stimulus in a global fashion may increase the probability that it is associated with the concept to be learned in memory. A study of the interaction of individual differences in global vs. analytic processing with concept acquisition and representation should provide information relevant to theories of cognitive structure as well as differences in processing capacities. A concept identification (CI) task using highly contextualized stimuli with many encoding possibilities is a potentially profitable task in the endeavor. Nahinsky, Oeschger, and O'Leary (1977) reported experiments that placed heavy emphasis upon influence of context in the concept acquisition process. In certain conditions, well-known individuals identified by salient descriptions were used as stimuli. Findings indicated strong tendencies toward a strategy involving global processing of stimuli, storing them as units, and contrasting them in memory en route to solution. Evidence suggested that such a strategy was effective only to the extent that all solution information was present on few enough trials to limit the memory load required by storage of individual stimuli.

Davis and Frank (1979) reviewed evidence that indicates that field independence is positively related to efficiency of concept learning, with less evidence supporting identifiable strategy differences related to the field independence variable. However, the results of Nahinsky, Morgan, and Oeschger (1979) suggest an interaction among strategy, efficiency, and important 
task parameters that leaves open questions of the nature of the operation of field independence in abstraction and disembedding.

Nahinsky et al. (1979) reported a CI experiment using contextualized stimuli of the type employed by Nahinsky et al. (1977). The acquisition task was followed by a surprise recognition memory test for the task stimuli. Subjects were tested on the embedded figures test (EFT) as a measure of field independence. Recognition performance revealed strong memory for specific task stimuli. Furthermore, memory for stimuli was significantly correlated with trial of last error (TLE), indicating that such episodic memory impeded the abstraction process. Task strategy differences were not highly correlated with EFT scores, although EFT was correlated with detailed aspects of recognition performance. Since certain aspects of such performance may reflect strategy as well as capacity differences, an understanding of the operation of field independence in the situation is quite incomplete.

We have reexamined results of the above experiment using a simple processing model to assess subjects' strategies. The model, to be described, applies to recognition latencies and relies upon ease of assessing contextual information relative to task-relevant information to detect strategies. By this approach we were able to separate strategy choice from field independence and examine how this cognitive variable interacts with such choice in information processing.

\section{METHOD}

\section{Subjects and Procedure}

The 48 subjects and the procedure used in obtaining the recognition latencies for assessing strategy were those described in Nahinsky et al. (1979). Subjects were first presented a twovalue conjunctive $\mathrm{CI}$ task in which they were to classify a series of 16 stimuli varied on four dimensions descriptive of people as positive or negative instances. Following the CI task subjects were instructed to perform an unannounced recognition task in which they were required to distinguish between the 16 problem stimuli and 16 new stimuli.

Each of the two problem series contained eight stimuli associated with names and pictorial exemplars of well known people and eight stimuli containing only verbal descriptions. Of the new stimuli, four contained verbal descriptions matching an old pictorial stimulus but associated with different pictorial exemplars, four pictorial stimuli contained verbal descriptions of a matched set of four old nonpictorial stimuli, four nonpictorial stimuli contained verbal descriptions of a matched set of four old pictorial stimuli, and four stimuli contained verbal descriptions not found on any old stimuli. Subjects were instructed that no information other than that from the four verbally descriptive values would be relevant to solution in CI, although they might somehow use the pictorial exemplars as learning cues. To assess recognition memory, subjects were told that they would see a series of stimuli to which a confidence rating expressing their degree of certainty about having seen (or not having seen) each stimulus should be assigned using a 7-point scale ranging from high confidence in "newness" at the low end to high confidence in "oldness" at the upper end.

Following the recognition memory task, subjects were administered Form V of the group EFT (Jackson, Messick, \& Myers, 1964).

\section{Processing Strategy Assessment}

A simple processing model was employed that assumed that recognition latencies would reflect the accessibility of taskirrelevant contextual information available from the CI task.

Suppose subjects approached the CI problem by storing available pictorial exemplars (e.g., Custer) and used them to redintegrate the verbal descriptive values (e.g., real, American, historical, soldier) in solving the problem. Such emphasis on distinctive encoding should lead to enhancement of recognition performance by pictorial context. It was thus assumed that "global" processors would first test for presence of familiar pictorial representation in episodic problem memory before testing verbal descriptions. A calculation of proportion of total variance $\left(\omega^{2}\right)$ in recognition latency attributable to the contrast between pictorial and nonpictorial stimuli was used as a measure of tendency to use a global strategy.

Subjects were rank ordered by $\omega^{2}$ values, with high values associated with global processing strategies and low values associated with verbal processing (no bias toward using pictorial stimuli). There were no instances in which a subject processed nonpictorial stimuli faster than pictorial stimuli on the average. The subjects were then split into groups by application of Fisher's clustering algorithm (Hartigan, 1975) for one-dimensional data to the ordered $\omega^{2}$ values. Subsequent analysis was then carried out with regard to these clusters.

\section{RESULTS}

The correlation between EFT and $\omega^{2}$ was nonsignificant $(r=.03)$, indicating that processing strategy chosen was independent of field independence. This result may account for the failure of Nahinsky et al. (1979) to find significant relationships between measures of task efficiency and field independence.

The results of Fisher's algorithm for marking maximum clusters are presented in Table 1. Since the proportion of variance accounted for by two partitions was greater than that for three partitions, subsequent analysis focused on the two-partition clusters. In assessing the relationship of EFT to the two experimental tasks, five measures were examined. Two measured from the CI task were obtained, TLE and the average latency to classify stimuli after the TLE $\left(\overline{\mathrm{L}}_{\mathrm{AL}}\right)$. Three recognition performance measures were included in the analysis. Individual recognition performance was measured by a transformation of rating data to a nonparametric signal detection measure, AG (Pollack, Norman, \& Galanter, 1964). Mean confidence ratings $(\overline{\mathrm{CR}})$ and average latency of confidence ratings $\left(\overline{\mathrm{L}}_{\mathrm{CR}}\right)$ were also measured.

Table 1

Proportion of Total Variance Accounted for by Pictorial vs. Verbal Processing Difference $\left(\omega^{2}\right)$ as a Function of Maximum Group Partitions

\begin{tabular}{lrccc}
\hline & $\mathrm{N}$ & Range of $\omega^{2}$ Mean $\omega^{2}$ & \multicolumn{1}{c}{$\mathrm{s}^{2}$} \\
\hline & \multicolumn{4}{c}{ Three Partitions $\left(\omega^{2}=.642\right)$} \\
Pictorial & 7 & $.60-.23$ & .337 & .169 \\
Indeterminate & 14 & $.23-.06$ & .136 & .042 \\
Verbal & 27 & $.05-.00$ & .018 & .022 \\
& \multicolumn{4}{c}{ Two Partitions $\left(\omega^{2}=.686\right)$} \\
Pictorial & 9 & $.60-.15$ & .313 & .156 \\
Verbal & 39 & $.14-.00$ & .049 & .051 \\
\hline
\end{tabular}


The correlation coefficient obtained from the 39 subjects in the predominantly verbal (lower) cluster between EFT and TLE approached significance $(\mathrm{r}=-.29, \mathrm{p}=.074)$. It is noteworthy that these same variables are related in the opposite direction for subjects in the predominantly pictorial cluster $(r=.59$, $p=.074)$. The difference between these two correlations approached significance $(z=1.84, p=.066)$. Since the number of subjects in the predominantly pictorial cluster was small $(\mathrm{N}=9)$ and the scores were variable, subsequent analyses concentrated on the assessment of strategy from the performance variables for subjects in the predominantly analytic cluster only. (The term analytic is used here to apply to the strategy in which subjects showed no tendency to differentially process pictorial stimuli. We infer that they abstracted the taskrelevant verbal descriptions from the total context.)

Correlation coefficients between recognition performance measures, CI measures, and EFT are given in Table 2.

A multiple correlation computed between the set of five task measures and EFT yielded a significant value $(\mathrm{R}=.530, \mathrm{p}=.045)$. The multiple correlation for these same measures with EFT for all 48 subjects, however, was not significant $(R=.411, p=.154)$. Table 2 shows the partial correlations of each of the five performance measures and EFT, holding the other four variables constant. The values show that TLE and AG account for the major share of the variance, with the correlation approaching conventional significance for TLE and reaching significance for $A G(p<.05)$. A multiple correlation between these two variables and EFT was then computed and was significant $(\mathrm{R}=.463, \mathrm{p}=.001)$. The difference between this correlation and that based upon all five variables was nonsignificant $[\mathrm{F}(3,33)=1.02]$, suggesting that the two variables were mainly responsible for the significant relationship between task variables and EFT. The resulting partial correlations with EFT, holding the other variables constant, were both significant (for TLE, $\mathrm{r}=.441, \mathrm{p}<.01$; for $\mathrm{AG}, \mathrm{r}=.381$, $\mathrm{p}<.02$ ).

It should be noted that the negative correlation between $\overline{\mathrm{L}}_{\mathrm{CR}}$ and EFT indicates a tendency for field

Table 2

Correlation Coefficients and Partial Correlations (PC) of Five Performance Measures with EFT

\begin{tabular}{lcccccc}
\hline & $\mathrm{TLE}$ & $\overline{\mathrm{L}}_{\mathrm{AL}}$ & $\overline{\mathrm{CR}}$ & $\overline{\mathrm{L}}_{\mathrm{CR}}$ & $\mathrm{AG}$ & $\mathrm{PC}$ \\
\hline $\mathrm{TLE}$ & & & & & & $-.290^{*}$ \\
$\overline{\mathrm{L}}_{\mathrm{AL}}$ & $.35^{* *}$ & & & & & -.003 \\
$\overline{\mathrm{CR}}$ & -.08 & -.05 & & & & -.239 \\
$\overline{\mathrm{L}}_{\mathrm{CR}}$ & $.65 \dagger$ & $.56 \dagger$ & -.10 & & & -.165 \\
$\mathrm{AG}$ & $.46 \dagger$ & .09 & -.12 & $.39^{* *}$ & & $.388^{* *}$ \\
$\mathrm{EFT}$ & $-.29^{*}$ & -.20 & -.21 & $-.26^{*}$ & .19 & \\
\hline
\end{tabular}

Note-PC $=$ correlation with EFT with other variables held constant. $\quad{ }^{*} p<.10 . \quad{ }^{* *} p<.05 . \quad t p<.01$. independence to be associated with more rapid access in the recognition task. The significant positive correlation between $\overline{\mathrm{L}}_{\mathrm{CR}}$ and $\mathrm{AG}$, however, indicates a speed-accuracy tradeoff that could mask the relationship between $\mathrm{AG}$ and EFT. The partial correlation between EFT and AG holding $\overline{\mathrm{L}}_{\mathrm{CR}}$ constant was significant $(\mathrm{r}=.328, \mathrm{p}=.024)$, a finding that supports this hypothesis.

\section{DISCUSSION}

When strategy choice was accounted for, there was a significant relationship between field independence and efficiency in abstraction and memory tasks. Contrary to evidence cited by Davis and Frank (1979) that field independence related to efficiency and not to identifiable strategies in CI, Nahinsky et al. (1979) found no strong evidence of a correlation with efficiency. Rather, they found a general tendency to respond to stimuli in a more global fashion. However, it should be noted that these investigators used highly distinctive stimuli with strong encoding possibilities in contast to stylized stimuli characteristic of most CI research. Insofar as such stimuli enhance possibilities of a global CI strategy of the sort suggested above, the relative contribution of strategy choice to the range of performance is increased. We have shown that strategy choice was independent of field independence, and such choices obscured the contribution of field independence to efficiency found by other investigators.

For those attempting analytic processing, field independence was related positively to both $\mathrm{CI}$ and recognition memory despite the negative relationship between CI efficiency (TLE) and recognition memory (AG). The latter relationship is compatible with the concept of a limited-capacity working memory in which there is a tradeoff between resources devoted to encoding/storage and hypothesis testing procedures. The former relationship then indicates that field-independent individuals have a superior working memory, either through more storage capacity or more efficient processing, or through some combination of the two.

It should be noted that superior recognition for field independents required ability to access pictorial information, the only discriminator between old and new stimuli. This superior performance prevailed despite a strategy that made processing of such information at most secondary. However, the superiority was revealed only when recognition latency was partialled out. Thus field-independent individuals stored more information, but the incidental nature of the information entailed a greater accessing effort, perhaps because of lesser priority in the storage structure.

Finally, implications for strategy-ability interactions should be considered. The results suggest that analytic ability is important to individuals only if they select analytic strategies despite inducements to do otherwise. Is it true that fielddependent individuals would do well to select global learning strategies? The limited data suggest this. At any rate, the lack of dependence of strategy choice on field independence suggests educational implications for matching individuals to processing strategies.

\section{REFERENCES}

Davis, J. K., \& Frank, B. M. Learning and memory of field independent-dependent individuals. Journal of Research in Personality, 1979, 13, 469-479.

Hartigan, J. Clustering algorithms. New York: Wiley, 1975. 
JACkson, D. N., Messick, S., \& Myers, C. T. Evaluation of group and individual forms of embedded-figures measures of field-independence. Educational and Psychological Measurement, 1964, 24, 177-192.

MacLeod, C. M., Hunt, E. B., \& Mathews, N. N. Individual differences in the verification of sentence-picture relationships. Journal of Verbal Learning and Verbal Behavior, 1978, 17, 493-507.

Nahinsky, I. D., Morgan, M. S., \& Oeschger, D. E. Cognitive strategies, field dependence, and the abstraction process. Journal of Research in Personality, 1979, 13, 490-504.
Nahinsky, I. D., Oeschger, D. E., \& O'Leary, D. Stimulus memory and contextual cues in the abstraction process. Canadian Journal of Psychology, 1977, 31, 102-112.

Pollack, I., Norman, D. A., \& Galanter, E. An efficient nonparametric analysis of recognition memory. Psychonomic Science, 1964, 1, 327-328.

Witkin, H. A., Dyk, R. B., Faterson, H. F., Goodenough, D. R., \& KArP, S. A. Psychological differentiation. New York: Wiley, 1962.

(Received for publication December 5, 1979.) 